\title{
TEMPERATURE JUMP IN DEGENERATE QUANTUM GASES IN THE PRESENCE OF A BOSE - EINSTEIN CONDENSATE
}

\author{
Anatoly V. Latyshev and Alexander Yushkanov \\ Department of Mathematical Analysis and Department of Theoretical Physics, \\ Moscow State Regional University, 105005, Moscow, Radio st., 10-A
}

(Dated: 10 ноября 2018 г.)

\begin{abstract}
We construct a kinetic equation modeling the behavior of degenerate quantum Bose gases whose collision rate depends on the momentum of elementary excitations. We consider the case where the phonon component is the decisive factor in the elementary excitations. We analytically solve the half-space boundary value problem of the temperature jump at the boundary of the degenerate Bose gas in the presence of a Bose - Einstein condensate.
\end{abstract}

Keywords: degenerate quantum Bose gas, collision integral, Bose - Einstein condensate, phonon component, temperature jump, Kapitsa resistance.

PACS numbers: 05.20.Dd Kinetic theory, 05.30.Jp Boson systems, 05.60.-k Transport processes, 03.75.Nt Other Bose - Einstein condensation phenomena

\section{INTRODUCTION}

The behavior of quantum gases has aroused increased interest in recent years. In particular, this is related to the development of experimental procedures for producing and studying quantum gases at extremely low temperatures [1]. The bulk properties of quantum gases have been studied in the majority of papers [2] and [3].

At the same time, it is obviously important to take boundary effects on the properties of such systems into account. We mention a paper where the thermodynamic equilibrium properties of quantum gases in a half-space were considered [4].

Along with the equilibrium properties, the nonequilibrium properties of quantum gases bounded in space attract interest. In particular, such a phenomenon as the temperature jump [5] at the interface between a gas and a condensed (in particular, solid) body in the presence of a heat flux normal to the surface is important. Such a temperature jump is frequently called the Kapitsa temperature jump [6].

We note that up to now, the Kapitsa jump has been calculated in the regime where only phonon scattering at the interface between two media was taken into account and phonon scattering in the bulk was neglected [7]. Here, we take namely the effect of scattering of elementary excitations of the Bose gas (phonons) in the bulk into account.
We take the character of phonon scattering by the surface into account by introducing a phenomenological coefficient of specular phonon scattering by the surface. This approach is thus additional to that proposed in [7].

In [8], we considered the temperature jump problem in a quantum Fermi gas. We obtained an analytic solution for an arbitrary degree of gas degeneracy. In [9], we considered a similar problem for a Bose gas. But the gas was assumed to be nondegenerate, i.e., we did not take the presence of a Bose - Einstein condensate into account.

This paper is devoted to analyzing the temperature jump problem in a degenerate Bose gas. The presence of a Bose - Einstein condensate leads to a considerable modification of both the problem statement and the solution method. In this case, to describe kinetic processes near the surface, we use a kinetic equation with a model collision integral. We assume that the boundary conditions at the surface are specular-diffuse.

\section{DERIVATION OF THE KINETIC EQUATION}

To describe the gas behavior, we use a kinetic equation with a model collision integral analogous to that used to describe a classical gas. We take the quantum character of the Bose gas and the presence of the Bose - Einstein condensate into account. For a rarefied Bose gas, the evolution of the gas particle distribution function $f$ can 
be described by the kinetic equation [5]

$$
\frac{\partial f}{\partial t}+\frac{\partial \varepsilon}{\partial \mathbf{p}} \nabla f=I[f]
$$

where $\mathcal{E}$ is the kinetic energy of gas particles, $\mathbf{p}$ is the gas particle momentum, and $I[f]$ is the collision integral.

In the case of the kinetic description of a degenerate Bose gas, we must take into account that the properties of the Bose - Einstein condensate can change as functions of the space and time coordinates, i.e., we must consider a two-liquid model (more precisely, a "two-fluid" model because we consider a gas rather than a liquid). We let $\rho_{c}=\rho_{c}(\mathbf{r}, t)$ and $\mathbf{u}_{c}=\mathbf{u}_{c}(\mathbf{r}, t)$ denote the density and velocity of the Bose - Einstein condensate. We then can write the expressions [7]

$$
\begin{gathered}
\mathbf{j}=\rho_{c} \mathbf{u}_{c}, \quad \mathbf{Q}=\frac{\rho_{c} u_{c}^{2}}{2} \mathbf{u}_{c}, \\
\Pi_{i k}=\rho_{c} u_{c i} u_{c k} .
\end{gathered}
$$

for the densities $\mathbf{j}, \mathbf{Q}$, and $\Pi_{i k}$ of the respective mass, energy, and momentum fluxes of the Bose - Einstein condensate (under the assumption that the chemical potential is zero). The conservation laws for the number of particles, energy, and momentum require that the relations

$$
\begin{gathered}
\nabla \mathbf{j}=-\int I[f] d \Omega_{B}, \\
\nabla Q=-\int \varepsilon(\mathbf{p}) I[f] d \Omega_{B}, \\
\nabla \Pi=-\int \mathbf{p} I[f] d \Omega_{B}
\end{gathered}
$$

be satisfied in the stationary case. Here,

$$
d \Omega_{B}=\frac{(2 s+1) d^{3} p}{(2 \pi \hbar)^{3}},
$$

$s$ is the molecule spin, $\mathcal{E}(\mathbf{p})$ is the energy, $\hbar$ is the Planck constant and $I(f)$ is the collision integral in Eq.

In what follows, we are interested in the case of motion with small velocities (compared with thermal velocities). We note that for the Bose - Einstein condensate, the quantities $\mathbf{Q}$ and $\Pi_{i k}$ depend on the velocity nonlinearly (they are proportional to the third and second powers of the velocity). Therefore, in the approximation linear in the velocity $\mathbf{u}_{c}$, the energy and momentum conservation laws can be written as

$$
\begin{gathered}
\int \varepsilon(\mathbf{p}) I[f] d \Omega_{B}=0, \\
\int \mathbf{p} I[f] d \Omega_{B}=0 .
\end{gathered}
$$

According to the Bogolyubov theory, the relation for the excitation energy $\mathcal{E}(p)$ holds for a weakly interacting Bose gas [5]

$$
\varepsilon(p)=\left[u_{0}^{2} p^{2}+\left(\frac{p^{2}}{2 m}\right)^{2}\right]^{1 / 2},
$$

where

$$
u_{0}=\left(\frac{4 \pi \hbar^{2} a n}{m^{2}}\right)^{1 / 2},
$$

$a$ is the scattering length for gas molecules, $n$ is the concentration, $m$ is the mass, and $\mathbf{p}$ is the momentum of elementary excitations. The parameter $a$ characterizes the interaction force of gas molecules and can be assumed to be small for a weakly interacting gas.

In our previous paper [1], we considered the case where the relation

$$
u_{0}^{2} \ll \frac{k T}{m}
$$

holds for sufficiently small $a$, where $k$ is the Boltzmann constant and $T$ is the temperature. In that case, the first term in the brackets in (2) can be neglected. The expression for the energy $\mathcal{E}(p)$ takes the same form as in the case of noninteracting molecules:

$$
\varepsilon(p)=\frac{p^{2}}{2 m} .
$$

We now consider the case where the phonon component dominates in (2), i.e., where

$$
T \ll \frac{m u_{0}^{2}}{k} .
$$

In this case, we obtain

$$
\mathcal{E}(p)=u_{0}|\mathbf{p}|=u_{0} p .
$$

according to relation (2). Consequently,

$$
\frac{\partial \mathcal{E}(\mathbf{p})}{\partial \mathbf{p}}=u_{0} \frac{\mathbf{p}}{p}
$$

When considering kinetic equation (1), by gas molecules, we must understand the elementary 
excitations of the Bose gas with energy spectrum (2). The character of the elementary excitations is manifested in the properties of the collision integral. As a collision integral in Eq. (1), we take its $\tau-$ approximation. Then the character of the elementary excitations is manifested in the dependence of the collision rate on the excitation momentum [9], 10], [11]- 13]

$$
\frac{\partial f}{\partial t}+u_{0} \frac{\mathbf{p}}{p} \frac{\partial f}{\partial \mathbf{r}}=\nu(\mathbf{p})\left(f_{B}^{*}-f\right) .
$$

Here, $f$ is the distribution function,

$$
\nu(\mathbf{p})=\nu_{0}\left|\mathbf{p}-\mathbf{p}_{0}\right|^{\gamma}
$$

is the dependence of the collision rate on the excitation momentum, and $\gamma$ is a constant. In the case where the phonon component dominates in the elementary excitations, $\gamma \geqslant 3$ [5]. We have $\mathbf{p}_{0}=m \mathbf{v}_{0}$, where $\mathbf{v}_{0}$ is the velocity of the normal component of the Bose gas, $f_{B}^{*}$ is the equilibrium function of the Bose - Einstein distribution

$$
f_{B}^{*}=\left[\exp \left(\frac{u_{0}\left|\mathbf{p}-\mathbf{p}_{*}\right|}{k T_{*}}\right)-1\right]^{-1},
$$

and $\nu_{0}$ is a model parameter having the meaning of the inverse mean free path $l, \nu_{0} \sim 1 / l$.

The parameters in $f_{B}^{*} / \mathrm{g}$, namely, $T_{*}$ and $\mathbf{u}_{*}$, can be determined from the requirement that the energy and momentum conservation laws

$$
\begin{gathered}
\int \nu(\mathbf{p}) \mathbf{p}\left[f-f_{B}^{*}\right] d^{3} p=0, \\
\int \nu(\mathbf{p}) \varepsilon(\mathbf{p})\left[f-f_{B}^{*}\right] d^{3} p=0
\end{gathered}
$$

be applicable. The conservation law for the number of particles is inapplicable here because of the transition of a fraction of particles to the Bose - Einstein condensate.

We now assume that the gas velocity is much less than the mean thermal velocity and the typical temperature variations along the mean free path $l$ are small compared with the gas temperature. Under these assumptions, the problem can be linearized.

We seek the distribution function in the form

$$
f=f_{B}^{s}(C)+\varphi(t, \mathbf{r}, \mathbf{C}) g(C),
$$

where

$$
f_{B}^{s}(C)=\frac{1}{\exp C-1},
$$

$\varphi$ is a new unknown function, $T_{s}$ is the surface temperature, $g(v)=-\frac{\partial}{\partial \varepsilon_{s}} f_{B}^{s}, \quad \varepsilon_{s}=C$, and we introduce the notation

$$
\mathbf{C}=\frac{u_{0} \mathbf{p}}{k T_{s}},
$$

and

$$
\varepsilon_{*}=\frac{u_{0}\left|\mathbf{p}-\mathbf{p}_{*}\right|}{k T_{*}} .
$$

We then have

$$
\begin{gathered}
f_{B}^{*}\left(\varepsilon_{*}\right)=\frac{1}{\exp \left(\varepsilon_{*}\right)-1}, \\
f_{B}^{s}(C)=\frac{1}{\exp C-1}, \\
g(C)=\frac{\exp C}{(\exp C-1)^{2}} .
\end{gathered}
$$

We linearize the local Bose - Einstein distribution (Bosean) $f_{B}^{*}$, passing to dimensionless quantities. We note that

$$
\begin{gathered}
\varepsilon_{*}=\frac{T_{s}}{T_{*}} \cdot \frac{u_{0}\left|\mathbf{p}-\mathbf{p}_{*}\right|}{k T_{s}}=\frac{T_{s}}{T_{s}+\delta T_{*}} \cdot \frac{u_{0}}{k T_{s}} \sqrt{\left(\mathbf{p}-\mathbf{p}_{*}\right)^{2}}= \\
=\left(1-\frac{\delta T_{*}}{T_{s}}\right) \frac{u_{0}}{k T_{s}}\left(p-\frac{\mathbf{p} \mathbf{p}_{*}}{p}\right),
\end{gathered}
$$

or

$$
\varepsilon_{*}=\left(1-\frac{\delta T_{*}}{T_{s}}\right)\left(C-\frac{\mathbf{C C}_{*}}{C}\right)=C-\frac{\mathbf{C C}_{*}}{C}-C \frac{\delta T_{*}}{T_{s}} .
$$

Therefore

$$
\delta \varepsilon_{*}=\varepsilon_{*}-\varepsilon_{s}=-\frac{\mathbf{C C}_{*}}{C}-C \frac{\delta T_{*}}{T_{s}},
$$

because $\varepsilon_{s}=C$.

Consequently,

$$
f_{B}^{*}=f_{B}^{s}+g(C)\left(\frac{\mathbf{C C}_{*}}{C}+C \frac{\delta T_{*}}{T_{s}}\right) .
$$

We note that the quantity $\nu(\mathbf{p})$ in Eq. (3) can be replaced with $\nu_{0} p^{\gamma}$ in the approximation under consideration.

We introduce the dimensionless time

$$
\tau=\nu_{0}\left(\frac{k T_{s}}{u_{0}}\right)^{\gamma} t
$$

and coordinate

$$
\mathbf{r}_{1}=\nu_{0}\left(\frac{k T_{s}}{u_{0}}\right)^{\gamma} \mathbf{r}
$$


It is now clear that Eq. (3) (in the dimensionless variables) becomes

$$
\frac{\partial \varphi}{\partial \tau}+\frac{\mathbf{C}}{C} \frac{\partial \varphi}{\partial \mathbf{r}_{1}}=C^{\gamma}\left[\frac{\mathbf{C C}_{*}}{C}+C \frac{\delta T_{*}}{T_{s}}-\varphi\right] .
$$

From the energy and momentum conservation laws, we find

$$
\begin{aligned}
\frac{\delta T_{*}}{T_{s}} & =\frac{1}{4 \pi g_{\gamma+4}} \int C^{\gamma+1} \varphi\left(\tau, \mathbf{r}_{1}, \mathbf{C}\right) g(C) d^{3} C, \\
C_{*} & =\frac{3}{4 \pi g_{\gamma+3}} \int C^{\gamma} \mathbf{C} \varphi\left(\tau, \mathbf{r}_{1}, \mathbf{C}\right) g(C) d^{3} C,
\end{aligned}
$$

where

$$
g_{\gamma+n}=\int_{0}^{\infty} C^{\gamma+n} g(C) d C, \quad n=0,1,2, \cdots .
$$

We represent Eq. (4) in the form that is standard in transport theory:

$$
\begin{gathered}
\frac{\partial \varphi}{\partial \tau}+\frac{\mathbf{C}}{C} \frac{\partial \varphi}{\partial \mathbf{r}_{1}}+C^{\gamma} \varphi\left(\tau, \mathbf{r}_{1}, \mathbf{C}\right)= \\
=\frac{1}{4 \pi} \int k\left(\mathbf{C}, \mathbf{C}^{\prime}\right) \varphi\left(\tau, \mathbf{r}_{1}, \mathbf{C}^{\prime}\right) g\left(C^{\prime}\right) d^{3} C,
\end{gathered}
$$

where $k\left(\mathbf{C}, \mathbf{C}^{\prime}\right)$ is the kernel of Eq. (5),

$$
k\left(\mathbf{C}, \mathbf{C}^{\prime}\right)=\frac{3}{g_{\gamma+3}} C^{\gamma-1} C^{\prime \gamma} \mathbf{C} \mathbf{C}^{\prime}+\frac{1}{g_{\gamma+4}} C^{\gamma+1} C^{\prime \gamma+1} .
$$

Equation (5) can be represented in the equivalent form

$$
\begin{gathered}
\frac{\partial \varphi}{\partial \tau}+\frac{\mathbf{C}}{C} \frac{\partial \varphi}{\partial \mathbf{r}_{1}}+C^{\gamma} \varphi\left(\tau, \mathbf{r}_{1}, \mathbf{C}\right)= \\
=\frac{3 C^{\gamma-1} \mathbf{C}}{4 \pi g_{\gamma+3}} \int C^{\prime \gamma} \mathbf{C}^{\prime} \varphi g\left(C^{\prime}\right) d^{3} C^{\prime}+ \\
+\frac{C^{\gamma+1}}{4 \pi g_{\gamma+4}} \int C^{\prime \gamma+1} \varphi g\left(C^{\prime}\right) d^{3} C^{\prime}
\end{gathered}
$$

\section{PROBLEM STATEMENT}

In the problem under consideration, a degenerate Bose gas occupies the half-space $x>0$ above a planar surface where the heat exchange between the condensed phase and the gas occurs. Therefore, the function $\varphi$ can be regarded as

$$
\varphi\left(\tau, \mathbf{r}_{1}, \mathbf{C}\right)=h(x, \mu, C)
$$

in what follows. Such a function satisfies the equation

$$
\begin{gathered}
\mu \frac{\partial h}{\partial x}+C^{\gamma} h(x, \mu, C)= \\
=\frac{1}{2} \int_{-1}^{1} \int_{0}^{\infty} K\left(\mu, C ; \mu^{\prime}, C^{\prime}\right) h\left(x, \mu^{\prime}, C^{\prime}\right) g\left(C^{\prime}\right) d \mu^{\prime} d C^{\prime},
\end{gathered}
$$

where $K\left(\mu, C ; \mu^{\prime}, C^{\prime}\right)$ is the kernel of Eq. (6),

$$
K\left(\mu, C ; \mu^{\prime}, C^{\prime}\right)=\frac{3 C^{\gamma-1} \mu C^{\prime \gamma+3} \mu^{\prime}}{g_{\gamma+3}}+\frac{C^{\gamma+1} C^{\prime \gamma+3}}{g_{\gamma+4}} .
$$

The problem is to find the value of the relative temperature jump

$$
\varepsilon_{T}=\frac{\Delta T}{T_{s}}
$$

where

$$
\Delta T=T_{s}-T
$$

as a function of $Q_{x}$, which is the projection of the heat flux on the $x$ axis. Taking linearity of the problem into account, we can write

$$
\varepsilon_{T}=R Q_{x}
$$

The dimensionless coefficient $R$ of the temperature jump is called the Kapitsa resistance.

It is obvious that Eq. (6) has the particular solutions

$$
h_{1}(x, \mu, C)=\mu \quad \text { and } \quad h_{2}(x, \mu, C)=C,
$$

and the Chapman - Enskog distribution function is

$$
h_{a s}(x, \mu, C)=B^{+} \mu-\varepsilon_{T} C,
$$

where the quantity $B^{+}$is proportional to the heat flux $Q_{x}$.

Assuming that the reflection of the elementary excitations from the wall is specular-diffuse, we now formulate the boundary conditions

$$
h(0, \mu, C)=q h(0,-\mu, C), \quad 0<\mu<1,
$$

$$
h(x, \mu, C)=
$$

$$
=B^{+} \mu-\varepsilon_{T} C+o(1), x \rightarrow+\infty,-1<\mu<0,
$$

where $q$ is the specular reflection coefficient.

The problem is to solve Eq. (6) with boundary conditions (7) and (8). Finding the value of the temperature jump $\varepsilon_{T}$ is of special interest. 


\section{REDUCTION TO THE INTEGRAL EQUATION}

We continue the function $\mathrm{h}$ to the half-space $x<0$ symmetrically:

$$
h(x, \mu, C)=h(-x,-\mu, C), \quad x<0 .
$$

For $x<0$, we then have

$$
h_{a s}(x, \mu, C)=B^{-} \mu-\varepsilon_{T} C,
$$

with $B^{+}=-B^{-}$.

We now separate the Chapman - Enskog distribution from the function $h$ assuming that

$$
h(x, \mu, C)=B^{ \pm} \mu-\varepsilon_{T} C+h_{c}(x, \mu, C) .
$$

For the function $h_{c}(x, \mu, C)$, we formulate the boundary conditions for the lower and upper half-spaces:

$$
\begin{gathered}
h_{c}(+0, \mu, C)= \\
=-(1+q) B^{+} \mu+(1-q) \varepsilon_{T} C+q h_{c}(+0,-\mu, C),
\end{gathered}
$$

where $\quad 0<\mu<1$,

$$
\begin{gathered}
h_{c}(-0, \mu, C)= \\
=-(1+q) B^{-} \mu+(1-q) \varepsilon_{T} C+q h_{c}(-0,-\mu, C),
\end{gathered}
$$

where $\quad-1<\mu<0$, and

$$
h_{c}(+\infty, \mu, C)=0, \quad h_{c}(-\infty, \mu, C)=0 .
$$

We include these boundary conditions in the kinetic equation. We obtain the equation

$$
\begin{gathered}
\frac{\mu}{C^{\gamma}} \frac{\partial h}{\partial x}+h_{c}(x, \mu, C)=\frac{C}{2 g_{\gamma+4}} W_{0}(x)+\frac{3 \mu}{2 g_{\gamma+3}} W_{1}(x)+ \\
+|\mu|\left\{-(1+q) B^{+}|\mu|+\right. \\
\left.+(1-q) \varepsilon_{T} C-(1-q) h_{c}(\mp 0, \mu, C)\right\} \delta(x)
\end{gathered}
$$

Here, $\delta(x)$ is the Dirac delta function, and

$$
\begin{gathered}
W_{m}(x)= \\
+\int_{-1}^{1} \int_{0}^{\infty} \mu^{m} C^{\gamma+3} h(x, \mu, C) g(C) d \mu d C, \quad m=0,1 .
\end{gathered}
$$

Equation (9) actually combines two equations. The point is that the term $h_{c}(-0, \mu, C)$ corresponds to positive $\mu: 0<\mu<1$ and the term $h_{c}(+0, \mu, C)$ corresponds to negative $\mu:-1<\mu<0$.

We seek the solution of Eqs. (9) in the form of Fourier integrals:

$$
h_{c}(x, \mu, C)=\frac{1}{2 \pi} \int_{-\infty}^{\infty} e^{i k x} \Phi(k, \mu, C) d k
$$

$$
\delta(x)=\frac{1}{2 \pi} \int_{-\infty}^{\infty} e^{i k x} d k
$$

$$
W_{0}(x)=\frac{1}{2 \pi} \int_{-\infty}^{\infty} e^{i k x} E_{0}(k) d k
$$

$$
W_{1}(x)=\frac{1}{2 \pi} \int_{-\infty}^{\infty} e^{i k x} E_{1}(k) d k
$$

Solving Eq. (10) with $x>0$ and $\mu<0$, assuming that the right-hand side of this equation is known, and assuming that the boundary conditions are satisfied far from the wall, we obtain

$$
h_{c}^{+}(x, \mu, C)=
$$

$$
=-\frac{C^{\gamma}}{\mu} \exp \left(-\frac{x}{\mu} C^{\gamma}\right) \int_{x}^{+\infty} \exp \left(\frac{t}{\mu} C^{\gamma}\right) W(t, \mu, C) d t
$$

where

$$
W(t, \mu, C)=\frac{C}{2 g_{\gamma+4}} W_{0}(t, \mu, C)+\frac{3 \mu}{2 g_{\gamma+3}} W_{1}(t, \mu, C) .
$$

After simple calculations, we obtain

$$
h_{c}^{+}(x, \mu, C)=\frac{C^{\gamma}}{2 \pi} \int_{-\infty}^{+\infty} \frac{e^{i k x} E(k, \mu, C) d k}{C^{\gamma}+i k \mu},
$$

where

$$
E(k, \mu, C)=\frac{C}{2 g_{\gamma+4}} E_{0}(k)+\frac{3 \mu}{2 g_{\gamma+3}} E_{1}(k) .
$$

We can similarly show that

$$
h_{c}^{-}(x, \mu, C)=\frac{C^{\gamma}}{2 \pi} \int_{-\infty}^{+\infty} \frac{e^{i k x} E(k, \mu, C) d k}{C^{\gamma}+i k \mu}
$$


From the last two expressions, taking the evenness of the function $E(k, \mu, C)$ with respect to the variable $k$ into account, we obtain

$$
\begin{gathered}
h_{c}^{ \pm}(0, \mu, C)= \\
=\frac{C^{\gamma}}{2 \pi} \int_{-\infty}^{+\infty} \frac{\left(C^{\gamma}-i k \mu\right) E(k, \mu, C) d k}{C^{2 \gamma}+k^{2} \mu^{2}}= \\
=\frac{C^{2 \gamma}}{\pi} \int_{0}^{+\infty} \frac{E(k, \mu, C) d k}{C^{2 \gamma}+k^{2} \mu^{2}} .
\end{gathered}
$$

Hence, we see that two Eqs. (9) can be combined into one:

$$
\begin{gathered}
\frac{\mu}{C^{\gamma}} \frac{\partial h}{\partial x}+h_{c}(x, \mu, C)= \\
=\frac{C}{2 g_{\gamma+4}} W_{0}(x)+\frac{3 \mu}{2 g_{\gamma+3}} W_{1}(x)+ \\
+|\mu|\left\{-(1+q) B^{+}|\mu|+\right. \\
\left.+(1-q) \varepsilon_{T} C-(1-q) h_{c}^{ \pm}(0, \mu, C)\right\} \delta(x) .
\end{gathered}
$$

We pass to the Fourier integrals in Eq. (12) and obtain the equation

$$
\begin{gathered}
\left(C^{\gamma}+i k \mu\right) \Phi(k, \mu, C)=\frac{C^{\gamma+1}}{2 g_{\gamma+4}} E_{0}(k)+\frac{3 C^{\gamma} \mu}{2 g_{\gamma+3}} E_{1}(k)+ \\
+C^{\gamma}|\mu|\left[-(1+q) B^{+}|\mu|+(1-q) \varepsilon_{T} C-C^{2 \gamma} \times\right. \\
\left.\times \frac{1-q}{\pi} \int_{0}^{\infty}\left[\frac{C E_{0}\left(k_{1}\right)}{2 g_{\gamma+4}}+\frac{3 \mu E_{1}\left(k_{1}\right)}{2 g_{\gamma+3}}\right] \frac{d k_{1}}{C^{2 \gamma}+k_{1}^{2} \mu^{2}}\right] .
\end{gathered}
$$

We consider equality (10). Rewriting it in terms of the Fourier integrals, we obtain

$$
\begin{gathered}
E_{j}(k)= \\
+\int_{-1}^{1} \int_{0}^{\infty} \mu^{j} \Phi(k, \mu, C) C^{\gamma+3} g(C) d \mu d C, \quad j=0,1 .
\end{gathered}
$$

We solve Eq. (13) for $\Phi(k, \mu, C)$ and substitute it in (14) for $j=0,1$. We obtain a system consisting of two characteristic equations. Without writing them, we combine them into one vector characteristic equation

$$
\begin{aligned}
\Lambda(k) E(k)= & -2(1+q) B^{+} T_{1}(k)+2(1-q) \varepsilon_{T} T_{2}(k)- \\
& -\frac{1-q}{\pi} \int_{0}^{\infty} J\left(k, k_{1}\right) E\left(k_{1}\right) d k_{1} .
\end{aligned}
$$

Here

$$
T_{m, l}(k)=\int_{0}^{1} \int_{0}^{\infty} \frac{\mu^{l} C^{m} g(C) d \mu d C}{C^{2 \gamma}+k^{2} \mu^{2}},
$$

$\Lambda(k)$ is the dispersion matrix

$$
\Lambda(k)=\left[\begin{array}{cc}
1-\frac{1}{g_{\gamma+4}} T_{3 \gamma+4,0}(k) & \frac{3 i k}{g_{\gamma+3}} T_{2 \gamma+3,2}(k) \\
\frac{i k}{g_{\gamma+4}} T_{2 \gamma+4,2}(k) & 1-\frac{3}{g_{\gamma+3}} T_{3 \gamma+3,2}(k)
\end{array}\right],
$$

$E(k)$ is an unknown column vector

$$
E(k)=\left[\begin{array}{c}
E_{0}(k) \\
E_{1}(k)
\end{array}\right],
$$

$T_{1}(k)$ and $T_{2}(k)$ are the column vectors of constant terms

$$
\begin{aligned}
& T_{1}(k)=\left[\begin{array}{c}
T_{3 \gamma+3,2}(k) \\
-i k T_{2 \gamma+3,4}(k)
\end{array}\right], \\
& T_{2}(k)=\left[\begin{array}{c}
T_{3 \gamma+4,1}(k) \\
-i k T_{2 \gamma+4,3}(k)
\end{array}\right],
\end{aligned}
$$

and $J\left(k, k_{1}\right)$ is the matrix function

$$
J\left(k, k_{1}\right)=\left[\begin{array}{cc}
\frac{1}{g_{\gamma+4}} J_{4 \gamma+4,1}\left(k, k_{1}\right) & -\frac{3 i k}{g_{\gamma+3}} J_{3 \gamma+3,3}\left(k, k_{1}\right) \\
-\frac{i k}{g_{\gamma+4}} J_{3 \gamma+4,3}\left(k, k_{1}\right) & \frac{3}{g_{\gamma+3}} J_{4 \gamma+3,3}\left(k, k_{1}\right)
\end{array}\right]
$$

which we call the Neumann matrix.

The integrals

$$
J_{m, l}\left(k, k_{1}\right)=\int_{0}^{1} \int_{0}^{\infty} \frac{\mu^{l} C^{m} g(C) d \mu d C}{\left(C^{2 \gamma}+k^{2} \mu^{2}\right)\left(C^{2 \gamma}+k_{1}^{2} \mu^{2}\right)}
$$

are introduced in the Neumann matrix. 


\section{METHOD OF SUCCESSIVE APPROXIMATIONS}

We seek the solution of Eq. (15) in the form

$$
\varepsilon_{T}=\frac{1+q}{1-q}\left[\varepsilon_{0}+\varepsilon_{1}(1-q)+\varepsilon_{2}(1-q)^{2}+\cdots\right]
$$

and

$$
\begin{gathered}
E(k)=2(1+q) \times \\
\times\left[E^{(0)}(k)+E^{(1)}(k)(1-q)+E^{(2)}(k)(1-q)^{2}+\cdots\right] .
\end{gathered}
$$

We substitute these equalities in the characteristic equation. We obtain the countable system of equations

$$
\begin{gathered}
\Lambda(k) E^{(0)}(k)=-B^{+} T_{1}(k)+\varepsilon_{0} T_{2}(k), \\
\Lambda(k) E^{(1)}(k)=\varepsilon_{1} T_{2}(k)-\frac{1}{\pi} \int_{0}^{\infty} J\left(k, k_{1}\right) E_{0}\left(k_{1}\right) d k_{1}, \\
\Lambda(k) E^{(2)}(k)= \\
+\varepsilon_{2} T_{2}(k)-\frac{1}{\pi} \int_{0}^{\infty} J\left(k, k_{1}\right) E_{1}\left(k_{1}\right) d k_{1}, \cdots .
\end{gathered}
$$

We transform the elements on the principal diagonal of the dispersion matrix:

$$
\begin{aligned}
& 1-\frac{1}{g_{\gamma+4}} T_{3 \gamma+4,0}(k)=\frac{k^{2}}{g_{\gamma+4}} T_{\gamma+4,2}(k), \\
& 1-\frac{1}{g_{\gamma+3}} T_{3 \gamma+4,2}(k)=\frac{3 k^{2}}{g_{\gamma+3}} T_{\gamma+3,4}(k) .
\end{aligned}
$$

The dispersion matrix function now becomes

$$
\Lambda(k)=\left[\begin{array}{ll}
\frac{k^{2}}{g_{\gamma+4}} T_{\gamma+4,2}(k) & \frac{3 i k}{g_{\gamma+3}} T_{2 \gamma+3,2}(k) \\
\frac{i k}{g_{\gamma+4}} T_{2 \gamma+4,2}(k) & \frac{3 k^{2}}{g_{\gamma+3}} T_{\gamma+3,4}(k)
\end{array}\right] .
$$

We call the determinant of the dispersion matrix the dispersion function:

$$
\lambda(z) \equiv \operatorname{det} \Lambda(z)=k^{2} \omega(k),
$$

where

$$
\omega(k)=\frac{3 k^{2}}{g_{\gamma+3} g_{\gamma+4}} \times
$$

$$
\times\left[k^{2} T_{\gamma+4,2}(k) T_{\gamma+3,4}(k)+T_{2 \gamma+3,2}(k) T_{2 \gamma+4,2}(k)\right] .
$$

The matrix inverse to the dispersion matrix is

$$
\Lambda^{-1}(k)=\frac{D(k)}{\lambda(k)},
$$

, where

$$
D(k)=\left[\begin{array}{cc}
\frac{3 k^{2}}{g_{n+3}} T_{n+3,4}(k) & -\frac{3 i k}{g_{n+3}} T_{2 n+3,2}(k) \\
-\frac{i k}{g_{n+4}} T_{2 n+4,2}(k) & \frac{k^{2}}{g_{n+4}} T_{n+4,2}(k)
\end{array}\right] .
$$

We consider the construction of series (17). We set

$$
E^{(0)}(k)=\left[\begin{array}{c}
E_{0}^{(0)}(k) \\
E_{1}^{(0)}(k)
\end{array}\right] .
$$

We consider the construction of series (17). We set

$$
\begin{gathered}
E^{(0)}(k)=2 \frac{D(k)}{\lambda(k)} \times \\
\times\left[\begin{array}{c}
-B^{+} T_{3 \gamma+3,2}(k)+\varepsilon_{0} T_{3 \gamma+4,1}(k) \\
i k\left(B^{+} T_{2 \gamma+3,4}(k)+\varepsilon_{0} T_{2 \gamma+4,3}(k)\right)
\end{array}\right] .
\end{gathered}
$$

Substituting expression (21) in Eq. (19), we find $E^{(1)}(k)$. Substituting $E^{(1)}(k)$ in Eq. (20), we then find $E^{(2)}(k)$. Continuing this process without bound, we construct all terms of series (17).

We now show how to construct the terms of series (16). For this, we write vector equality (21) in the form of two scalar equalities:

$$
E_{0}^{(0)}=\frac{1}{k^{2} \omega(k)}\left\{-\frac{3 k^{2}}{g_{\gamma+3}} T_{\gamma+3,3}(k) \times\right.
$$$$
\times\left[B^{+} T_{3 \gamma+3,2}(k)-\varepsilon_{0} T_{3 \gamma+4,1}(k)\right]+
$$

$$
\left.+\frac{3 k^{2}}{g_{\gamma+3}} T_{2 \gamma+3,2}(k)\left[B^{+} T_{2 \gamma+3,4}(k)+\varepsilon_{0} T_{2 \gamma+4,3}(k)\right]\right\}
$$

$$
E_{1}^{(0)}=\frac{1}{k^{2} \omega(k)} \times
$$

$$
\times\left\{i k T_{2 \gamma+4,2}(k)\left[B^{+} T_{3 \gamma+3,2}(k)-\varepsilon_{0} T_{3 \gamma+4,1}(k)\right]+\right.
$$

$$
\left.+i k^{3} T_{\gamma+4,2}(k)\left[B^{+} T_{2 \gamma+3,4}(k)+\varepsilon_{0} T_{2 \gamma+4,3}(k)\right]\right\} .
$$


It can be seen from expression (22) that the function $E_{0}^{(0)}(k)$ has no singularities at zero, while the function $E_{1}^{(0)}(k)$ according to (23) has a second-order pole at $k=$ 0 . Eliminating the singularity at zero, we obtain

$$
\varepsilon_{0}=B^{+} \frac{T_{3 \gamma+3,2}(0)}{T_{3 \gamma+4,1}(0)}=B^{+} \frac{2 g_{\gamma+3}}{3 g_{\gamma+4}} .
$$

The quantity $B^{+}$is proportional to the heat flux:

$$
\mathbf{Q}=\int f(x, \mathbf{p}) \frac{\partial \varepsilon(p)}{\partial \mathbf{p}} \varepsilon(p) d \Omega_{B} .
$$

We transform this expression as

$$
\mathbf{Q}=\frac{(2 s+1) u_{0}^{2}}{(2 \pi \hbar)^{3}} \int h(x, \mathbf{p}) g(p) \mathbf{p} d^{3} p .
$$

Integrating in this expression over the dimensionless momentum, we obtain

$$
\mathbf{Q}=\frac{(2 s+1)\left(k T_{s}\right)^{4}}{(2 \pi \hbar)^{3} u_{0}^{2}} \int h(x, \mathbf{C}) g(C) \mathbf{C} d^{3} C .
$$

We replace here the function $h(x, \mathbf{C})$ with its Chapman - Enskog expansion $h_{a s}(x, \mathbf{C})$. As a result, we have

$$
\begin{aligned}
& Q_{x}=\frac{(2 s+1)\left(k T_{s}\right)^{4}}{(2 \pi \hbar)^{3} u_{0}^{2}} \int_{-1}^{1} \int_{0}^{\infty} \int_{0}^{2 \pi}\left[\varepsilon_{T} C+B^{+} \mu\right] \times \\
& \times g(C) C^{3} \mu d \mu d C d \chi=\frac{(2 s+1)\left(k T_{s}\right)^{4}}{(2 \pi \hbar)^{3} u_{0}^{2}} \cdot \frac{4 \pi}{3} g_{3} B^{+}
\end{aligned}
$$

for the $x$ component of the heat flux.

We hence have

$$
B^{+}=\frac{6 \pi^{2}}{g_{3}} \cdot \frac{\hbar^{3} u_{0}^{2} Q_{x}}{(2 s+1)\left(k T_{s}\right)^{4}} .
$$

The quantity $\varepsilon_{0}$ is therefore

$$
\varepsilon_{0}=\frac{4 \pi^{2} g_{\gamma+3}}{g_{3} g_{\gamma+4}} \cdot \frac{\hbar^{3} u_{0}^{2}}{(2 s+1)\left(k T_{s}\right)^{4}} Q_{x} .
$$

Returning to the formula for the temperature jump

$$
\Delta T=R Q_{x},
$$

we find from expression (24) that the Kapitsa resistance in the zeroth approximation is

$$
R=\frac{4 \pi^{2} g_{\gamma+3}}{g_{3} g_{\gamma+4}} \cdot \frac{1+q}{1-q} \cdot \frac{\hbar^{3} u_{0}^{2}}{(2 s+1) k^{4} T_{s}^{3}} .
$$

We rewrite this equation in the form

$$
R=C(\gamma, q) \cdot \frac{\hbar^{3} u_{0}^{2}}{(2 s+1) k^{4} T_{s}^{3}},
$$

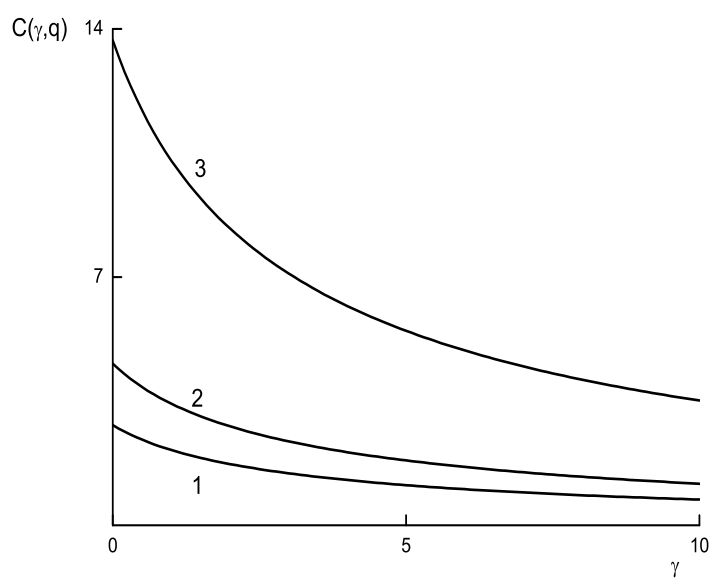

Fig. 1. Dependence of the temperature jump coefficient on the parameter $\gamma$ : the specular reflection coefficient is $q=0.3$ for curve $1, q=0.5$ for curve 2 , and $q=0.8$ for curve 3 .

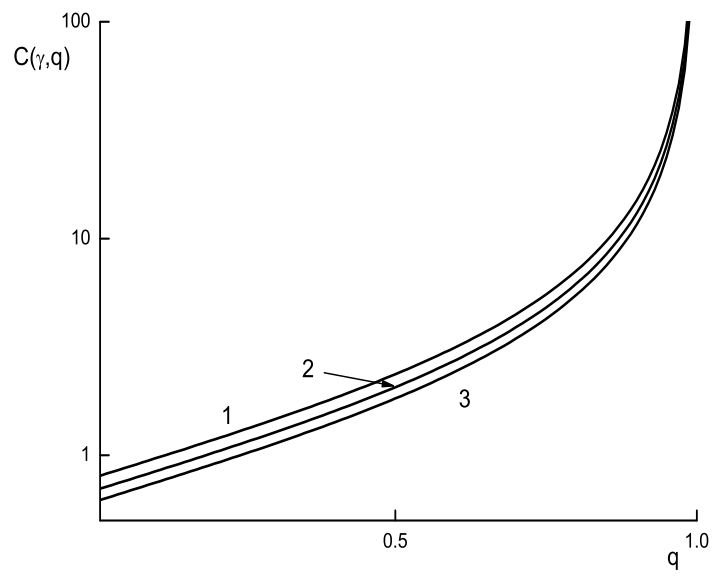

Fig. 2. Dependence of the temperature jump coefficient on the specular reflection coefficient q: the collision parameter is $\gamma=3$ for curve $1, \gamma=4$ for curve 2 , and $\gamma=5$ for curve 3 .

where

$$
C(\gamma, q)=\frac{4 \pi^{2} g_{\gamma+3}}{g_{3} g_{\gamma+4}} \cdot \frac{1+q}{1-q}
$$

is the (dimensionless) coefficient of the temperature jump.

The plus sign in formula (25) indicates that the wall temperature is higher that the phonon component temperature. 
The graphs of the behavior of the temperature jump coefficient as a function of the parameter $\gamma$ and the specular reflection coefficient $q$ are shown in Figs. 1 and 2.

It can be seen from these graphs that the quantity $C(\gamma, q)$ decreases monotonically as the parameter $\gamma$ increases. As the specular reflection coefficient $q$ tends to unity, the quantity $C(\gamma, q)$ increases without bound because the heat exchange between the wall and the gas adjoining it becomes impossible in this limit.

\section{CONCLUSIONS}

We have constructed a kinetic equation for a degenerate quantum Bose gas whose collision rate depends on the momentum of elementary excitations of the Bose gas. We considered the case where the phonon component is the key factor in the elementary excitations. The boundary conditions were assumed to be speculardiffuse. We solved the half-space boundary value problem of the temperature jump at the boundary of a degenerate gas in the presence of a Bose - Einstein condensate. We derived a formula for finding the temperature jump and calculating the Kapitsa resistance.

We have developed a sufficiently general method for solving kinetic equations with specular-diffusive boundary conditions; this method was first proposed in [14], where the problem of the skin effect was considered.

[1] L. P. Pitaevskii, Phys. Usp., 49, 333-351 (2007).
[2] H. Spon, Kinetics of the Bose-Einstein Condensation, arXiv:0809.4551 1 [cond-mat.mes-hall] (2008).

[3] Hai Pang, Wu-Sheng Dai, Mi Xie, J. Phys. A. Mat. Gen. 39 (2006), 2563-2571; arXiv:cond-mat/0603289 (2006).

[4] L. Samaj and B. Jancovici, ArXiv: cond-mat/0701773.

[5] E. M. Lifshitz and L. P. Pitaevskii, Statistical Physics, Part 2 [in Russian], Nauka, Moscow (1978); English transl.: Statistical Physics: Part 2. Theory of the Condensed State (Vol. 9 of Course of Theoretical Physics, L. D. Landau and E. M. Lifshits, eds.), Pergamon Press, Oxford (1980).

[6] Yu. V. Prokhorov, ed., Encyclopedic Dictionary of Physics [in Russian], Bol'shaya Rossiiskaya Entsiklopediya, Moscow (1955).

[7] I. M. Khalatnikov, An Introduction to the Theory of Superfluidity [in Russian], Nauka, Moscow (1965); English transl., Addison-Wesley, Redwood City, Calif. (1989).

[8] A. V. Latyshev and A. A. Yushkanov, Theor. Math. Phys., 134(2), 271-284, (2003).

[9] A. V. Latyshev and A. A. Yushkanov, Mathematical Modeling, 15(5), 80-94, (2003).

[10] Carlo Cercignani, Theory and Application of the Boltzmann Equation, Scottish Academic, Edinburgh (1975).

[11] A. V. Latyshev and A. A. Yushkanov, Theor. Math. Phys., 155(3), 498-511, (2008).

[12] A. V. Latyshev and A. A. Yushkanov, Theor. Math. Phys., 162(1), 95-105, (2010).

[13] A. V. Latyshev and A. A. Yushkanov, Williamstype Kinetic Equations and Their Exact Solutions [in Russian], Moscow State Regional Univ., 2004.

[14] A. V. Latyshev and A. A. Yushkanov, Comp. Mathem. and Mathem. Phys., 49(1), 131-145, (2009). 